\section{INFLAMMATION}

\section{D6 clears away inflammatory chemokines}

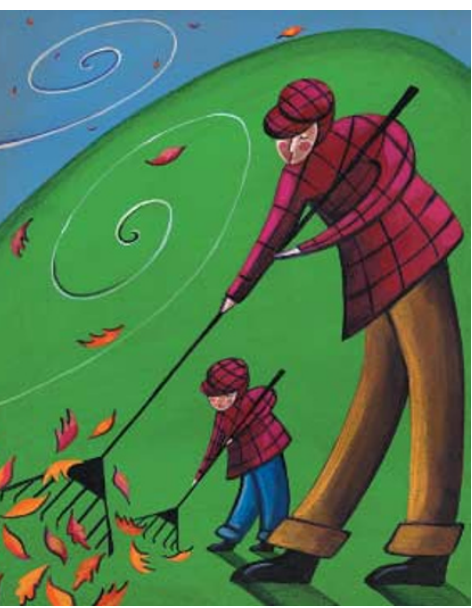

Although much is known about the initiation of pro-inflammatory responses, relatively little is known about how these responses are resolved. In a recent report in Nature Immunology, Thomas Jamieson et al. show that the chemokine receptor D6 is involved in clearing $\beta$-chemokines from inflamed skin and therefore in resolving inflammation.

D6 is a seven-membrane-spanning receptor that binds pro-inflammatory members of the $\beta$-chemokine family, such as CC-chemokine ligand 2 (CCL2; also known as MCP1) and CCL3 (also known as MIP1 $\alpha$ ), but it does not bind constitutively expressed $\beta$-chemokines, such as CCL19 and CCL21. However, unlike other chemokine receptors, D6 does not seem to signal after ligand binding but, instead, internalizes its ligands and targets them for degradation, indicating that D6 might function as a decoy receptor. So, to test the in vivo functions of D6, the authors generated D6-deficient mice and analysed their responses in models of cutaneous inflammation. At 8 and 18 hours after the induction of inflammation in the skin by the phorbol ester 12-O-tetradecanoylphorbol-13-acetate (TPA), the concentrations of CCL2 and CCL3 were similar in the skin of both D6-deficient and wild-type mice. However, by 24 hours, the concentrations of these chemokines were significantly higher in the D6-deficient mice, which is consistent with a role for D6 in the post-inflammatory clearance of $\beta$-chemokines from the skin. By contrast, levels of the non-D6binding chemokine CXC-chemokine ligand 2 (CXCL2; also known as MIP2) did not differ between wildtype and D6-deficient mice at any time point.

The authors next asked whether the increased concentrations of residual pro-inflammatory $\beta$-chemokines had pathological consequences. Indeed, although inflammation in wild-type mice induced by 3 applications of TPA resolved after 4 days, D6-deficient mice developed skin pathology that was characterized by epidermal hyperproliferation and inflammatory-cell infiltration similar to the pathology of human psoriasis. They showed that this process was dependent on tumournecrosis factor (TNF), as TNF-specific antibodies completely abrogated the

ANTIGEN PRESENTATION

\title{
A new route to cross-presentation
}

MHC class I molecules usually present peptides that are derived from endogenous antigens. But they can also present peptides that are derived from exogenous antigens, through a process known as crosspresentation. Various mechanisms have been proposed to explain how this occurs. Now, Joost Neijssen and colleagues propose a novel mechanism - which they have named gapjunction-mediated immunological coupling (GMIC) — to add to the list.

Gap junctions are channels that form between adjacent cells. Each cell contributes a hemichannel of six connexin molecules to the functional gap junction. These channels allow the passive exchange of ions, nutrients and signalling components between cells. Haematopoietic cells express connexin-43, so the authors set out to investigate the role of gap junctions in peptide transfer between cells and whether this could contribute to cross-presentation.

First, the authors looked at whether peptides can be transferred between cells through gap junctions. The human squamous-cell carcinoma line A431, which does not form gap junctions, was stably transfected with connexin-43, resulting in the formation of gap junctions. Non-degradable, fluorescently labelled peptides were introduced into these cells, and peptide transfer was analysed by confocal microscopy. This showed that such transfer was possible and could be blocked using gap-junction inhibitors. The rate of peptide transfer was also determined and was shown to be inversely proportional to the size of the peptide: that is, the transfer rate was decreased for longer peptides.

Because the cytoplasm contains several cytosolic peptidases, the authors next looked at the impact of these peptidases on peptide transfer between cells. Using quenched peptides that become fluorescent after degradation, they found that cytosolic peptidases can limit, but not prevent, the spread of peptides from cell to cell. So, does this peptide transfer have immunological relevance? To test this, the authors expressed an influenza-derived peptide in connexin-43-transfected A431 cells, which do not express HLA-A2, and they cultured these cells with HLA-A2-transfected A431 cells. When HLA-A2-restricted T cells specific for the influenza-derived peptide were added to the culture, a T-cell response was detected, showing that peptide transfer had occurred through gap junctions. Next, the authors carried out similar experiments using primary human monocytes. Connexin-43transfected A431 cells were infected with influenza, and after 16 hours, viral propagation was inhibited. These infected cells were then cultured with primary human monocytes that had been stimulated to express connexin- 43 by exposure to interferon- $\gamma$ and tumour-necrosis factor. The infected cells were loaded with a dye such that monocytes that had obtained peptides through gap junctions were detectable. Only dye-containing monocytes were found to stimulate a cytotoxic T-lymphocyte response.

These findings indicate a novel mechanism for cross-presentation that does not require release of intracellular antigens from cells. Interestingly, Langerhans cells and intestinal dendritic cells have many gap-junction contacts with surrounding cells, and monocytes can form gap junctions in response to 'danger' signals. In addition, gap junctions are inactivated in many tumour cells, indicating that this is another mechanism by which tumour cells might avoid detection. Apoptotic bodies can be crosspresented to $\mathrm{CD}^{+} \mathrm{T}$ cells, but in situations in which cells are prevented from undergoing apoptosis, cross-presentation could be achieved by GMIC.

(0) References and links Elaine Bell ORIGINAL RESEARCH PAPER Neijssen, J. et al. Cross-presentation by intercellular peptide transfer through gap junctions. Nature 434, 83-88 (2005) 
psoriasiform pathology. Further analysis of the inflammatory infiltrate indicated that $\mathrm{T}$ cells and mast cells accumulated at high levels in the inflamed skin of D6-deficient mice but not in that of wild-type mice. Infiltration of T cells into the epidermis was partially responsible for the subsequent accumulation of dermal mast cells, which contribute to development of the pathology through their release of granule products.

So, D6 functions in vivo as a decoy receptor, clearing away residual $\beta$-chemokines in inflamed skin, thereby helping to avoid aberrant recruitment of inflammatory cells and subsequent pathology.

Lucy Bird

(2) References and links ORIGINAL RESEARCH PAPER Jamieson, T. et al. The chemokine receptor D6 limits the inflammatory response in vivo. Nature Immunol. 6, 403-411 (2005)

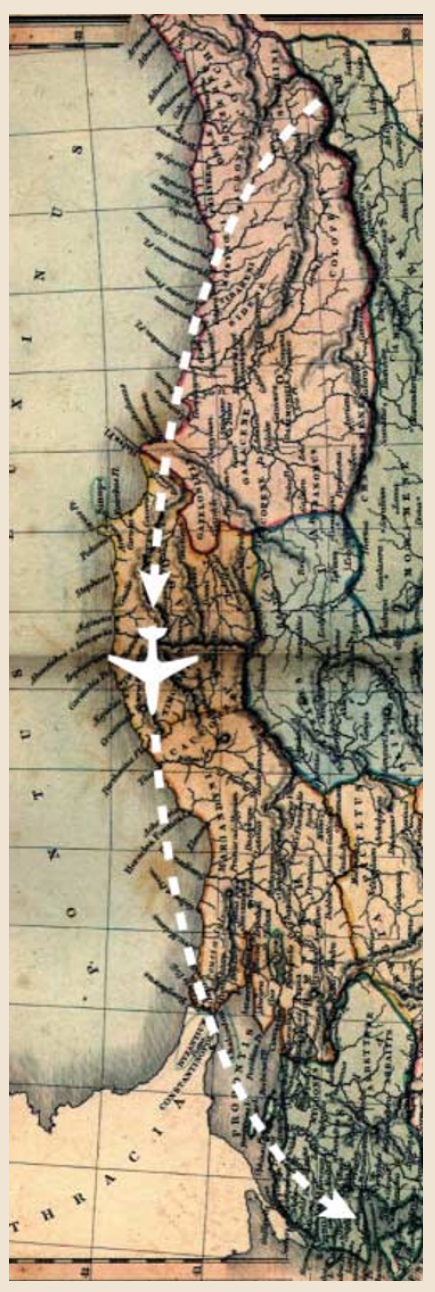

THYMIC DEVELOPMENT

\section{TRAF6 distributes tolerance}

Medullary thymic epithelial cells (mTECs) are important for the negative selection of thymocytes. However, the molecular regulation of mTEC differentiation and organization within the thymus is not well understood. New insight into this process has now been provided by a study published in Science, which shows that tumour-necrosis-factor receptor (TNFR)associated factor 6 (TRAF6) is required for the differentiation and organization of mTECs and for the induction of T-cell self-tolerance.

TRAF6 is a cytoplasmic adaptor protein that transduces signals from several members of the TNFR superfamily, leading to the activation of transcription factors such as activator protein 1 (AP1) and nuclear factor- $\kappa \mathrm{B}(\mathrm{NF}-\kappa \mathrm{B})$. Previous studies from Jun-ichiro Inoue's group have shown that the thymi of TRAF6-deficient mice are atrophied, leading the researchers to hypothesize that TRAF6 is important for thymic organogenesis. In this new study, the medulla was shown to be smaller in thymi from TRAF6deficient mice than in thymi from wild-type animals, and the corticomedullary junction was ill defined. mTECs were present in the Traf $6^{-1}$ thymi, but they were dispersed and not clustered in the medulla; they also failed to bind Ulex europaeus agglutinin-1 (UEA-1), a lectin that binds mature mTECs.

In addition to impaired maturation, TRAF6deficient mTECs also expressed reduced levels of Aire mRNA, which encodes a protein that induces expression of tissue-specific antigens (TSAs) such that developing thymocytes are tolerized to these TSAs in the thymus. Consistent with this, TRAF6deficient mice developed inflammation in several organs, including the lungs, liver and pancreas. Such inflammation in TRAF6-deficient mice was probably not only a result of decreased expression by mTECs of mRNA encoding TSAs but also a result of the reduced numbers of $\mathrm{CD} 4{ }^{+} \mathrm{CD} 25^{+}$ regulatory $\mathrm{T}\left(\mathrm{T}_{\text {Reg }}\right)$ cells. Similar inflammatory infiltrates were observed when TRAF6-deficient thymi depleted of haematopoietic cells were transplanted into nude mice, indicating that an intrinsic non-haematopoietic-cell defect was responsible for the autoimmune phenotype of TRAF6-deficient mice.

The phenotype of TRAF6-deficient mice was similar to that observed in mice lacking the NF- $\kappa B$-family member REL-B. So, the authors examined fetal thymic stroma isolated from Traf $6^{-1-}$ mice and found decreased levels of REL-B in these cells compared with wild-type fetal thymic stroma. Furthermore, when TRAF6

\section{was complemented in TRAF6-deficient} mTEC lines, REL-B levels increased. Additional support for the idea that TRAF6 induces Rel-b transcription is provided by the presence of two NF- $\mathrm{KB}$ - and putative AP1-binding sites (transcription factors that are known to be activated downstream of TRAF6 signalling) in the Rel-b promoter.

This study identifies TRAF6 as a crucial molecular component of the signalling pathway that regulates $\mathrm{mTEC}$ differentiation and organization in the thymus. TRAF6 is also important in the development of self-tolerance, and future studies will focus on how TRAF6induced REL-B regulates expression of Aire and development of $\mathrm{T}_{\mathrm{Reg}}$ cells. Interestingly, the phenotype of TRAF6-deficient mice is also similar to that of mice lacking NF- $\kappa \mathrm{B}$-inducing kinase (NIK), which regulates the proteasomal processing of the NF- $\kappa B$-family member 100 to generate the REL-B-binding partner p52. Understanding the relationship between NIK- and TRAF6-regulated activation of NF- $\kappa B$-family members will help to define the molecular control of thymic organogenesis and central tolerance.

Karen Honey

(2) References and links

ORIGINAL RESEARCH PAPER Akiyama, T. et al. Dependence of self-tolerance on TRAF6-directed development of thymic stroma. Science 10 Feb 2005 (doi:10.1126/science1105677)

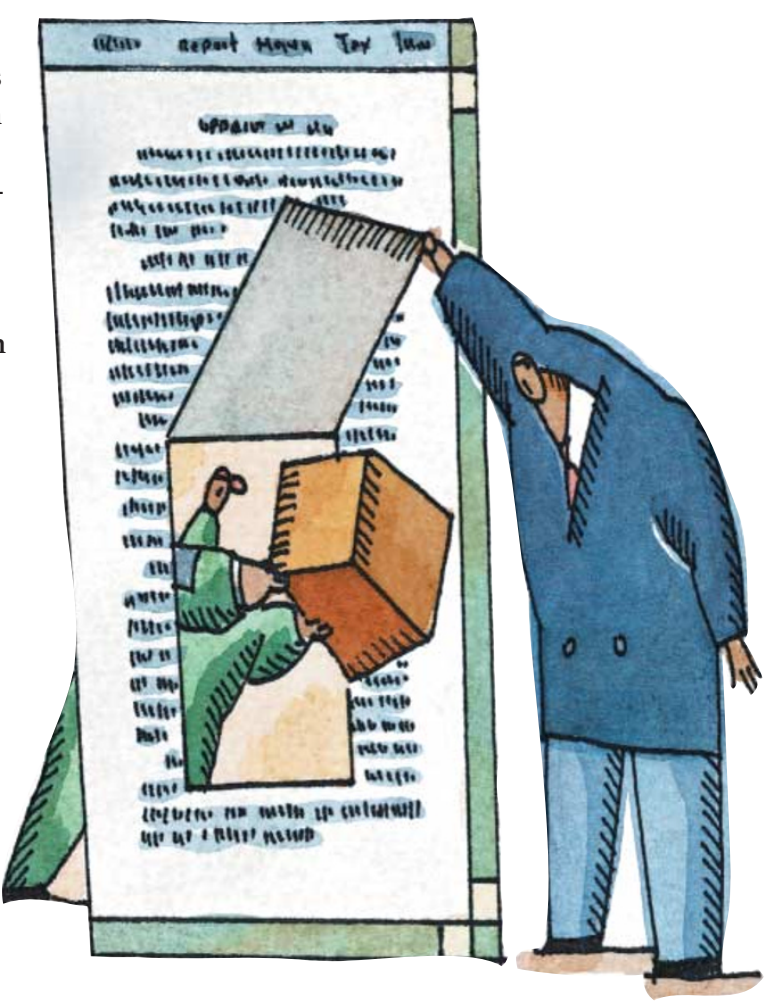

